\section{iettsomian aflectures}

\author{
ON
}

\section{MIDWIFERY AND DISEASES OF WOMEN.}

Delivered before the Medical Society of London. BY

\section{H. F. ROUTH, M.D.,}

PHYSICIAN TO THE SAMARITAN HOSPITAI, FOR WOMEN AND CHILDREN.

\section{Lecture II (concluded).}

Ox some Ponsts having Reference to The

Differential Diagnosis of Fibrous

'TUMOURS OF THE UTERUS.

Ovarian Diseases. It is with ovarian cases that the diagnosis is often very difficult. The differential symptoms on which most reliance is usually placed are the following. Ovarian tumours generally arise from one side; fluctuation is felt in them; the shape of the tumour is symmetrical and pyriform; the hand can be passed between the parietes of the abdomen low down and the tumour; and the uterus has a shorter cavity. Now, I believe that every one of these symptoms may be present, and the tumour after all prove to be a fibroid.

1. The ovarian tumour grows from one side. This may be true in many instances; but fibrous tumours are not unusally found to begin on one side. I have met several such, more particularly where they contain fluid or are situated in the latter walls. Moreover, in the case of pediculated tumours and pelvian bodies, to which I have before referred, these tumours are commonly found on one side.

2. Fluctuation may also be absent in ovarian disease. Though the tumour be ovarian, it may be fibrous. such a case occurred to Ir. Murphy; and was successfully operated upon by Mr. Erichsen.

Two cases where this occurred are also mentioned by M. Liègeois. (Bull. Soc. An., xxix, pp. 140-41.) In the first, there was found after death a large tumour of the size of a fist in each ovary, of a fibrous nature. Microscopically, both exhibited fibro-plastic characters. In the second case, there was a large fibrous tumour weighing eight pounds. Another case is mentioned by M. Icery (Ib., xxviii, p. 135), where a large uterine fibroid co-existed with fibroids of both ovaries.

But we may have a sensation of fluctuation in a fibroid produced by four causes : $a$. A comparatively soft, fleshy, flabby condition, quite irrespective of fluid ; $b$. Unusual vascularity; $c$. The presence of odema; $d$. Uterine false or real cysts, as before seen.

a. The following is an example of the first variety, where an extrauterine fibroid, and withal solid, fluctuated, and was, besides, diagnosed to be ovarian. It is related by M. Bourcy. (Bulletin Soc. Anat., xxx, p. 160.)

A woman, aged 45, was admitted into the Hôpital Cochin, under M. Gosselin. She had had children, and was in good health up to two years back. On admission, the symptoms were, tumefaction and pain of the abdomen since the cessation of the catamenia ten or twelve months previously. The pains had become greatly worse during the last two months; they were very severe, like labour-pains. Micturition and de- fæcation were very difficult. She had kept her bed two months, and was only able to stand and walk by using a belt. Abdominal palpation detected a hard, non-fluctuating tumour of the size of a fœtal head, bilobed, larger on the right side. There was an indolent hard tumour in the right breast. Vaginal examination revealed the tumour at the bottom of the vagina. No cervix was to be found (which led to the belief that the tumour was external to the uterus). This patient, after suffering agonising pains, only relieved by opium, and losing flesh rapidly, died two months after admission.

A post mortem examination was made twenty-four hours after death. Palpation made through the abdomen revealed fluctuation. On opening it, the intestines were found to be pushed upwards and backwards, and were contracted in diameter. A large fibrous tumour, rounded, much larger than an adult head, was situated behind the uterus, between it and the rectum, which it compressed behind. The tumour was encysted; it might have been enucleated. The cyst envelope was hard, thick, fibrous, and closely attached to the posterior part of the uterus. The tumour itself, when cut, appeared marbled, here and there tinged with ecchymosis, varying in consistence, friable ; percussion of it produced tremblings $(t \cdot e m-$ blotements) in the mass. The union with the uterus was remarkable. It was attached firmly to the tumour, and on the right side. The cervix was thinned and elongated. The body, the curvature of which was preserved, was normal, firmly adherent to the enveloping membrane. On a level with the union of this membrane and the posterior face of the uterus, there was a notable thickening, a hard tissue, fatty, and intermixed with some osteo-calcareous productions. The uterine appendages were healthy. The kidneys were large, and the ureters dilated, by reason of the pressure of the tumour, to the size of the small intestine.

A more remarkable case still was that of Koelerle's.

It was one of enormous tumour, weighing above sixty-six pounds, which, before the operation, was, by himself as well as others, found to fluctuate. "The sensation of the wave of liquid seemed so evident, that no one could doubt at all that fluid existed in it. This fluctuation was most evident on the left side, and gentle taps could be transmitted through it very clearly to twenty, and even thirty, centimetres." On tapping before the operation, only blood exuded. During the operation, when the abdominal parietes were cut through and the tumour exposed, "at the slightest tap of the finger, the tumour undulated as a mass of jelly, or like a bladder incompletely filled with fluid"; and yet this tumour turned out to be a solid fibrous tumour.

In both these instances, fluctuation was so manifest during life, that a mistake was certain to be made. 'The great test, however, of the transmission of the aortic sounds, was not tried.

$b$. The second variety of deceptive fluctuation, from excessive vascularity, is more common; and I have met several examples of it. They are eminently the vascular fibroids. Mr. Brown lately mentioned an example of this kind in his lecture, where a fibroid was complicated by pregnancy; and, no doubt, the false fluctuation here felt was due to this cause. In another case, also of Mr. Brown's, the particulars of which he has kindly forwarded to me, this same feeling of fluctuation existed ; and here, also, an exploratory incision was made. When the tumour was cut into, it bled profusely, and then was found to be made up of a number of engorged vessels, 
The patient ultimately did well. When so experienced an operator as Mr. Brown is deceived, you may imagine the difficulty of a correct diagnosis in these cases. Yet I think they may often be made out. The souffle, amounting almost to a splenic murmur, sometimes accompanied by a thrill, in them betrays their vascularity. An exploratory puncture will bleed very freely. and often require the actual cautery to be applied to arrest its flow; but if it be small, and made through the vagina, it is not attended, as I have seen, with any objectionable results.

I saw a case of the same kind, which Dr. Greenhalgh kindly shewed me at St. Bartholomew's, in which also this pseudo-fluctuation existed, and where an exploratory puncture was followed by excessive bleeding, which was checked only by the actual cautery ; and I have met other cases.

$c$. The third cause of fluctuation is nedema of fibrous tumours, and it is a symptom which is far more common than is generally believed. I have met with it several times. Once or twice it has appeared to me to have been induced in a previously solid tumour by the very examinations made to diagnose its nature. Occasionally, it has originated from a blow.

In these instances, although fluctuation may be absent, the feel is so elastic that you cannot believe it to be solid. And what adds to the difficulty is, that the single or double cardiac sounds before referred to may be destroyed. Here the previous history, and it may be an exploratory puncture, is the best means of diagnosis.

$d$. It is, however, with fibroids containing cysts, or fibro-cystic tumours, that the greater difficulty lies. The following example, given by M. Holaire (Bull. Soc. Anat., xxvii, p. 319), and which occurred to M. Rostan, is interesting in illustrating this error of diagnosis.

A woman, aged 46, two years ago, began to enlarge in the abdomen. The tumour began on the right side. At first slow in development, its growth gradually became more active, and she was admitted into the hospital. Rostan diagnosed ovarian disease, and tapped her in the right side. A yellowish urinecoloured fluid came away. The belly now became straight again. No tumour could be felt by palpation. She was much relieved, and no bad consequences followed this puncture. The abdomen was found to enlarge again. She was tapped again, with the same relief. But this was only temporary; for soon the abdomen began again to enlarge, and a tumour could now be felt, which, in a short time, grew to the size of a child's head. Digestive troubles followed. Icterus made its appearance, and marasmus. Finally, she died.

The post mortem examination revealed adhesions in the abdomen and partial peritonitis. The broad ligaments, ovaries, and Fallopian tubes, were all healthy. It was the uterus which was the seat of several encysted multilocular tumours, varying from the size of an orange to that of eggs and smaller still, in various degrees of development. There was no cancerous juice. Microscopically, M. Verneuil discovered only fibrous tissue of new formation. It was, therefore, most probably a recurrent fibroid.

I have already alluded to several of these cases in speaking of the softening of fibroids by cystic transformation. The great majority of these were mistaken for ovarian disease, and treated accordingly. I myself have known two instances, at least, where very large fibroids contained much fluid. The dia- gnosis in the one was correct; that in the second, altogether at fault.

It is in cases like these that the points to which I have before referred, in the necessity of knowing. fully the previous history and making a most minute examination, becomes so important. It is here, also, that, short of an exploratory incision, it may be wise to make use of an exploratory needle. 'The remarkable epithelial bodies and compound cells found in ovarian cysts, and the absence of fibrous débris, will at once indicate that we have to do with an ovarian cyst and not a fibroid. This is an important point, and one which I believe has not been before sufficiently dwelt upon. More inquiries, microscopical as well as chemical, are needed to enable us accurately to tell the difference between fluid contained in a fibroid and that contained in an ovarian tumour. Years ago, Dr. Hughes Bennett did do so. In a case submitted to him, he was able to say that the tumour was fibroid in character.

There are two other symptoms upon which I wish to dwell, in connexion with the diagnosis of ovarian disease and fibroids. One is the state of the catamenial function; the other, referred to by Dr. M'Clintock, is derived from the position of the tumour and the inner wall of the anterior part of the abdomen. The disturbance of the catamenial function is not believed to afford much indication in the diagnosis of ovarian dropsy as opposed to the presence of fibroids. Mr. Brown says :

"That we may have irregularity of the menses, an excessive flow, or dysmenorrhœa; but suppression is rare. Nevertheless, we may not be able to discover any such catamenial derangements; and menstruation may have been regular throughout the disease, or become so after its definite establishment. Likewise, suppression attends the development of cancerous disease; and is common where a cyst rapidly developes, or where there has been a large drain of its serous contents. Kiwisch says the arrest of menstruation by the latter causes mentioned, is more frequent in compound than in simple cystic disease ; and that, as a symptom, it is not without its value in diagnosis and prognosis." (Brown on Ovarian Dropsy, p. 29.)

In bringing these opinions to the statistical test, I obtained the following results. In 150 cases, selected from my own experience and from that of others, and including several cases kindly furnished to me by Dr. Greenhalgh, I have noted the state of the catamenial function.

In 64 , or 42.6 per cent., it was regular. In 44 , or 36.6 per cent., it was arrested; but this number is too high; for in seven only was the suppression directly traceable to the development of the tumour. In 17 again, the women were about 45 , at which age the catamenia cease normally.

In 14 , or 9.2 per cent., menorrhagia or profuse menstruation existed.

In 16 , the catamenia were irregular; but 3 were about 45 years of age, and 4 had always had irregular menstruation; the percentage under this head would, therefore, be about 8 per cent.

3 women had never menstruated ; 2 had dysmenorrhœa; and 9 had scanty menstruation.

In most cases, therefore, if we exclude extrauterine fibroids, the differences in this function are sufficiently broad materially to exclude error. The state of menorrhagia is normally that of fibroids: quite exceptionally that in ovarian disease. 
Dr. M'Clintock states that, where we have an uterine tumour, the hand, say in its ulnar edge, cannot be passed down but to a very small extent between the internal abdominal wall and the tumour. In ovarian tumours, the ulnar edge of the hand can be passed deeply between them. However unpleasant it is to differ from so high an authority, I am compelled to say that my experience cannot confirm this statement. This is particularly the case in those examples where the uterus is lengthened by the fibrous growth and the bladder pushed up with it. Wherever, also, in an ovarian case, or even in an ordinary abdominal tumour, there are adhesions, the test fails. But, even without such adhesions, and in ovarian tumours, it cannot be done.

In conclusion, I must say that there are a class of cases which are still more puzzling; I mean those examples of fibroid coexisting with ovarian disease and solid ovarian tumours. I have collected notes of several such cases.

The case before quoted of M. Bouchet (Bull. Soc. An., xxviii, p. 10) was an instance of a fibroid of the uterus with ovarian cysts. M. Jury's case (ib., p. 135) was also an example of fibroids in both uterus and ovaries. M. Jules Simm (ib., 2nde liv., iii, p. 69) has recorded a case of fibrous tumour complicated with hernia and with ovarian cysts. This patient was operated upon for hernia. An artificial anus was the result, of which, with gangrene of the parts, the patient died.

The post mortem examination revealed an uterus studded on its whole surface with fibrous tumours, from the size of a lentil to that of an apple. One of these was encysted, and contained an unctuous yellow liquid. The uterine cavity was the shape of the letter $S$. The two ovaries were replaced by two cysts of the size of an egg. Another case is mentioned by M. Bouchet ( $i b$., xxix, p. 136), in which two enormous fibroids, one on each side of the uterus, larger than the fists, interparietal, existed, and the right ovary contained a cyst as large as the two other tumours conjoined.

Without question, the diagnosis in such cases is very difficult; nor have I anything now to advance on this point.

University College. The Atkinson Morley Surgical Scholarship has been awarded to Mr. Alexander Bruce, of London. The scholarship is of the value of $£ 45$ per annum, for three years.

The Exodus of Medical Officers from India. The Bengal Hurkaru states that a large number of the most distinguished of the medical officers of the late East India Company are leaving India.

Small-Pox in Berlin. The small-pox is at present raging in Berlin, and carrying off numerous victims every week. A panic has begun to seize the population on this account, and every morning the public medical institutions are besieged by crowds of adults anxious to be revaccinated, as well as by infants in the arms of their nurses who are to be subjected to the process for the first time.

Cape Coast Casualties. Returns just issued shew that amongst the many deaths of soldiers which have occurred on this fatal coast-of sixteen officers-are the names of Assistant-Surgeons Rutherford and Hooper. And amongst twenty-two officers invalided home are Surgeon Skeen, Assistant-Surgeon Sandison, Assistant-Surgeon Crow, Assistant-Surgeon Oughton, and Assiatant-Surgeon Lewis.

\section{Original Commumatations.}

\section{THE THERAPEUTICS OF CONSUMPTION.}

By Richard Payne Cotton, M.D.; Fellow of the Royal College of Physicians, London; Physician to the Hospital for Consumption and Diseases of the Chest, Brompton.

[Read before the Medical Society of London.]

Thene are few maladies in which Medicine has borne so variable a reputation as in consumption. We have heard over and over again of "specifics" for this disease ; and over and over again has the honest but disappointed physician expressed his conviction that phthisis is scarcely, if at all, under his control.

In the present communication, I shall endeavour to show that whilst, on the one hand, we may confidently assert that at present, at least, we have no specific remedy for consumption, we may, on the other hand, as confidently assert that our materia medica offers a number of auxiliaries of more or less value and importance in the treatment of this disease.

With a view to determine experimentally the comparative value of different therapeutic agents in consumption, I resolved, some five or six years ago, to prescribe certain substances to a given number of my hospital in-patients, taking care that such cases were free from all acute symptoms, as well as from any particular complications requiring special treatment. Each of the following substances was thus administered to twenty-five patients, this number having been chosen as sufficient, perhaps, for the purpose, and as possessing the advantage of at once showing a percentage simply by multiplying by four; viz., phosphorus; liquor potassæ; hydrochloric acid ; iodide of iron; iodide of potassium; chloride of sodium; vinum ferri ; glycerine ; sesquichloride of iron; chlorate of potassa; quinine; phosphoric acid. The experiments were published from time to time in the Medical Times and Gazette; but hitherto they have not been compared with each other, neither has any attempt been made at a general conclusion.

Here I may as well anticipate one or two very possible objections to my experiments. Some may consider that the number of cases was too small to show any satisfactory result; whilst others may suggest that there could not have been sufficient similarity in the cases themselves to render the several conclusions trustworthy. To the first objection I would reply, that it has taken me five years to observe the effects of these twelve substances upon the number of cases I determined upon; and as there is a limit not only to life, but also to the time and capacity for working out such observations, I felt it was impracticable to angment the number of patients for each experiment. And to the second objection I would remark, that, as it is no inconsiderable difficulty to discover even two consumptive persons strictly alike in all their symptoms, the attempt to find twenty-five parallel cases would have been fruitless. I may, however, at once state that $I$ am desirous that $m$ y experiments should be taken only for what they are worth. They do not profess to be absolutely conclnsive, but are simply intended as certain additions to that general stock of therapeutical facts which, when put together and thoroughly sifted, may, some day or other, lead to the discovery of important truths.

Phosphorus in many cases proved a good tonic and stimnlant, increasing both the appetite and strength 